\title{
High Sensitivity Refractive Index Sensor Based on Highly Overcoupled Tapered Fiber Optic Couplers
}

\author{
M. V. Hernández-Arriaga, M. Bello-Jiménez, A. Rodríguez-Cobos, R. López-Estopier and M. V. \\ Andrés, Member, IEEE
}

\begin{abstract}
In this work a simple and compact fiber optic sensor based on an overcoupled tapered fiber coupler is studied. The coupler is fabricated to be operated well beyond the initial coupling cycles, where the rapid exchange of energy between outputs ports enable the fabrication of a highly sensitive device. The suitability and sensitivity of the proposed scheme is demonstrated by measuring refractive index (RI) variations of sugar concentrations in water. The device presents a linear response in terms of power transmission or wavelength shift versus RI changes. The best achieved sensitivity is 0.442 units of normalized transmission per unit of sugar concentration, with a noise detection limit of 0.003 weight percent of sugar concentration (wt \%). From this result the minimum detectable RI change is estimated as $5 \times 10^{-6}$ refractive index unit (RIU). The sensor can be also wavelength-encoded, exhibiting a sensitivity of $2,171 \mathrm{~nm} / \mathrm{RIU}$, maintaining a linear response in a large range of RI. These experimental results are within the best results reported in the framework of fiber couplers and modal interferometers based RI sensors.
\end{abstract}

Index Terms-Biconical fiber coupler, refractive index sensor, spectral response.

\section{INTRODUCTION}

$\mathrm{F}$ IBER-OPTIC sensors with high sensitivity and resolution have been the subject of extensive investigations for the measurement of diverse physical and chemical parameters; these parameters include temperature [1]-[3], stress [4]-[6], curvature [7]-[9], and refractive index (RI) [10]-[13]. Among them, we find that fiber coupler based sensors are promising structures since they do not require expensive and complex fabrication procedures, assuming they are fabricated using a standard fusion and pulling technique. Fiber couplers provide two complementary outputs ports that enable a straightforward normalization, i. e. the ratio between the difference and the

This work has been financially supported by CONACyT grant 222476 .

M. V. Hernández, M. A. Bello, and A. Rodríguez, are with the Instituto de Investigación en Comunicación Óptica (IICO), Universidad Autónoma de San Luis Potosí, Av. Karakorum 1470 Lomas 4a Secc., 78210 San Luis Potosí, S.L.P, México.

R. López-Estopier is with CONACyT at the Instituto de Investigación en Comunicación Óptica (IICO), Universidad Autónoma de San Luis Potosí, Av. Karakorum 1470 Lomas 4 secc., C. P. 78210, San Luis Potosí, SLP, México. M. V. Andrés is with the Departamento de Física Aplicada y Electromagnetismo, ICMUV, Universidad de Valencia, C/Dr. Moliner 50, Burjassot, 46100 Valencia, Spain. addition, which compensates for power fluctuations, Beside this, also provide the advantages of compactness, high sensitivity, low-cost, in situ measurement and immunity to external electromagnetic interference. In this kind of sensors the coupler transmission response undergoes a shift in wavelength that it is strongly affected by the RI of the surrounding medium due to the evanescent field that is generated along the coupling region [14]. This effect was proposed and demonstrated in early works to develop fiber based refractometers [14], [15]. In recent years, optical sensors based on microfiber directional couplers have been proposed and demonstrated to perform high sensitivity measurements in many practical applications [16-23]. For the specific case of fiber coupler based RI sensors, these include fused biconical fibers [24]-[26], microfibers [16], [18], [27]-[30], photonic crystal fibers (PCF) [31]-[33], and specially designed two-core optical fibers [34], [35]. Most of these sensors are presented as wavelength codified and the achieved sensitivities are in the range from 1,125 to 30,100 nanometer $(\mathrm{nm})$ per refractive index unit (RIU), with a detection limit in a range from $4 \times 10^{-4}$ to $5 \times 10^{-7} \mathrm{RIU}$, respectively. Amplitude codified sensors have been also reported to provide high resolution [31], [36], [37], with a detection limit between $2 \times 10^{-5}$ and $4 \times 10^{-6}$ RIU. However, the sensors with the highest sensitivities and best resolutions are based on PCF and require selective infiltration of holes with the liquid under test, which imposes severe limitations from a practical point of view.

For practical applications, amplitude codified sensors are of particular interest since they do not require special equipment to determine small variations of the refractive index. To date, several authors have exploited the power transmission dependence to develop a fiber coupler based refractometer [14], [15], [31], [37]. Nevertheless, in those works the authors did not study the conditions for an optimal performance of the reported device. In a previous publication we reported a fused biconical fiber coupler (FBFC) structure for the detection of small variations of RI [36]. In that scheme the coupler transmission was analyzed to find a power transmission response that allows a linear relation between RI changes and the output signal. The coupler was operated within the firsts coupling cycles, and the minimum detectable RI change was estimated as $2.4 \times 10^{-5}$ RIU. Now in this paper our purpose is to report an improved FBFC scheme that is operated in a regime well beyond the initial coupling cycles; in order to improve the coupler sensitivity. The coupler is fabricated to 
reach the limits of the experimental implementation. Our study covers the analysis of the sensor in terms of normalized power variations and wavelength shifts; i. e., as amplitude and/or wavelength encoded sensor. In fact, by combining wavelength and power measurements our proposal exhibits large RI range with high resolution. Our experimental results are within the best results reported in the framework of fiber coupler based RI sensors and provide useful information for future optimization of the sensor response. Furthermore, our proposal preserves the simplicity and robustness required for practical applications.

\section{FUSED FIBER COUPLER FABRICATION}

The couplers are fabricated using a standard fusion and pulling technique [38], in which two single-mode optical fibers (Corning SMF-28) are twisted and fixed together on two translational motorized stages, as shown in Fig. 1(a). A gas mixture of oxygen and butane produces a flame which heats the fibers to make them moldable. The flame sweeps back and forth just below the twisted section of the fibers, and simultaneously the fibers are pulled from both ends fusing and tapering the fibers together. At the end of the process the coupler is composed of two biconical tapered transitions and a uniform taper waist. For monitoring the coupler fabrication, a tunable laser source emitting at $1550 \mathrm{~nm}$ is connected to port 1 $\left(P_{1}\right)$ of the fiber coupler, and the transmitted light is measured by two photodiodes, each one connected at output ports $3\left(P_{3}\right)$ and $4\left(P_{4}\right)$ of the fiber coupler. The transmitted signal is monitored in real time with a standard oscilloscope during the pulling process. In principle, by a precise control of the pulling length it is possible to fabricate a coupler with a specific coupling ratio. The power variation as a function of the pulling length, the so-called pulling signature, is shown in Fig. 1(b) at the cross-coupled port $\left(P_{4}\right)$. Fig. 1(b) gives the basic information to determine the required pulling length for the fabrication of a specific fiber coupler. In our experiments, the couplers are fabricated with two tapered fibers, each one with exponential transitions, a uniform taper waist of about 2 $\mathrm{mm}$, and a total length of about $20 \mathrm{~mm}$. In this specific case reported in Fig. 1, the final overall diameter of the coupler waist is $1 \mu \mathrm{m}$. The parameters of the fiber coupler are designed with a well-stablished model for fiber tapers [39].

With the objective of improving significantly previous results and trying to push the technique to the limits, we focus our attention in the fabrication of highly overcoupled fiber couplers. The intention is to operate the coupler in cycles well beyond to the initial coupling cycles in order to increase the coupler sensitivity. One characteristic at this regime is the rapid exchange of energy between outputs ports as a function of the pulling length. For illustration of this effect, the inset in Fig. 1(b) shows the exchange of power between outputs ports at the pulling length between 19.95 and $20.05 \mathrm{~mm}$; around 7 coupling cycles occur in a $0.1 \mathrm{~mm}$ interval of pulling length. Therefore, the fabrication of overcoupled couplers with a specific coupling ratio is not feasible due to the high degree of accuracy that would be required. To overcome this problem, a tunable laser source is included in our experimental scheme to enable a precise selection of the coupling ratio by tuning the wavelength, once the fabrication procedure has concluded. Fig. 1(c) shows the transmission spectrum of the coupler at the end of the pulling length process ( $23 \mathrm{~mm}$ of elongation), the coupler has a coupling ratio of 15:85 in air at the wavelength of $1550 \mathrm{~nm}$. However, by tuning the wavelength it is possible to select a desired coupling ratio. For example, a 50:50 coupling is achieved at a wavelength of $1546 \mathrm{~nm}$. The inset in Fig. 1(c) shows a microscope image of the coupler crosssection at the uniform taper waist. As it can be noted, the coupler has a weakly fused cross-section (two touching circular cylinders) [40]-[42], although the device exhibits a high coupling due to strong evanescent fields. In order to insure a robust and safely manipulable device, we limited our self to couplers with a $5 \mu \mathrm{m}$ waist cross-section in the rest of this work.
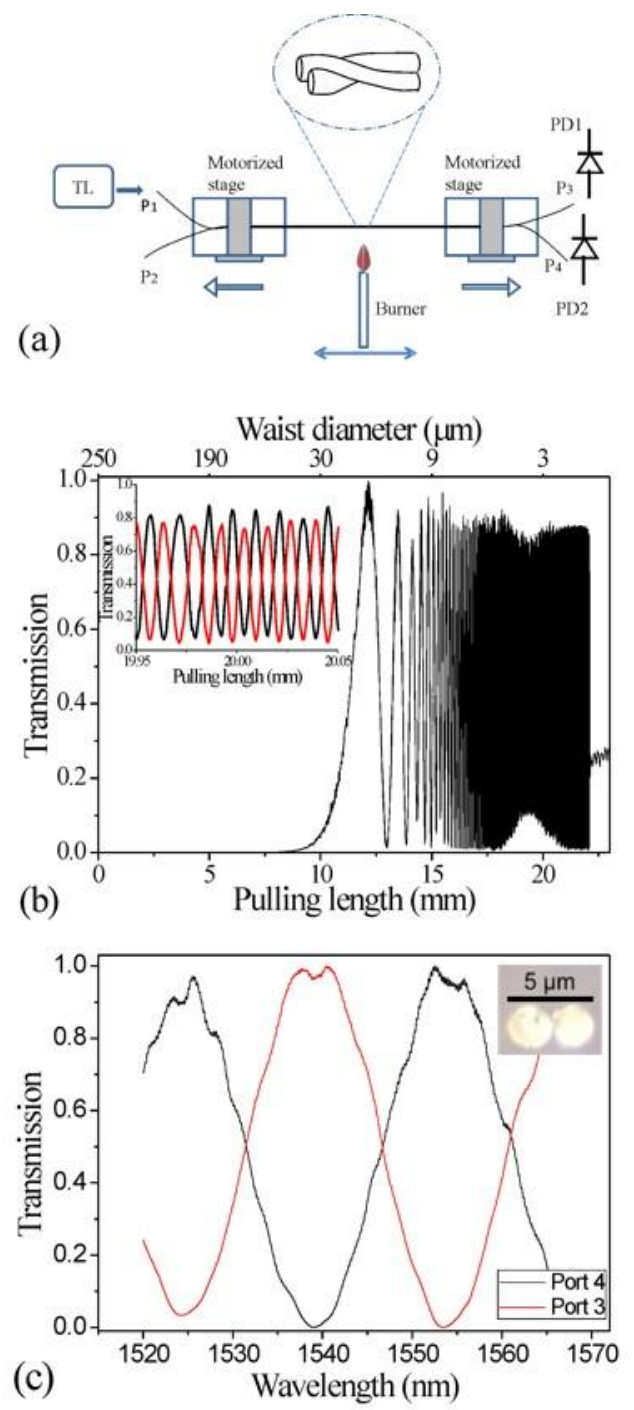

Fig. 1. Fabrication of overcoupled fused biconical fiber couplers. (a) Fabrication setup. (b) Power variation as a function of the pulling length. The inset shows the exchange of power between outputs ports for a pulling length between 19.95 and $20.05 \mathrm{~mm}$. (c) Transmission spectrum of the fused fiber coupler at the end of the pulling process ( $23 \mathrm{~mm}$ of elongation). The inset in (c) shows an image of the weakly fused cross-section with $5 \mu \mathrm{m}$ in width. 


\section{EXPERIMENTAL MEASUREMENTS AND DISCUSSION}

The experimental setup for measuring RI changes is illustrated in Fig. 2(a). The coupler is mounted on a microscope slide and fixed from both ends, so that the coupler waist is suspended in air to avoid contact with the glass. The sensor arrangement consist of a tunable laser diode connected to port 1 of the fiber coupler. The tunable source covers the wavelength range 1520 - $1570 \mathrm{~nm}$ and makes possible, by wavelength tuning, the selection of a desired coupling ratio. The transmission of light is measured by two photodiodes $P D 1$ and $P D 2$, each one connected at output ports 3 and 4 , respectively. These complementary outputs ports enable a straightforward normalization, i.e. the ratio between the difference and the addition, which compensates for power fluctuations. The transmission response is monitored with a standard oscilloscope during the experimental procedure.

The sensor characterization is carried out by measuring RI variations of sugar concentrations in water. A drop of a sugar solution is deposited onto the waist of the coupler, producing a shift of the transmission spectrum and a change of the coupling ratio at a given wavelength. To illustrate the measurement process, Fig. 2(b) shows a numerical simulation of the normalized coupler transmission as a function of the external RI. For simulations we consider a 4-mm uniform waist of $5 \mu \mathrm{m}$ in width composed of two weakly fused optical fibers, each one with a $2.5 \mu \mathrm{m}$ in diameter. Under these conditions the coupling coefficient is approximated by [43]:

$$
C=\frac{(2 \Delta)^{1 / 2}}{r} \frac{U^{2}}{V^{3}} \frac{K_{0}(W d / r)}{K_{1}^{2}(W)},
$$

where $r$ is the radius of the fiber cladding, $d$ is the center-tocenter separation between fibers, and $K_{0}$ and $K_{l}$ are the modified Bessel functions of second kind of order 0 and 1 . The parameters $U, V, W$, and $\Delta$ are defined as,

$$
\begin{aligned}
& U=r\left(k^{2} n_{2}^{2}-\beta^{2}\right)^{1 / 2}, \\
& V=k r\left(n_{2}^{2}-n_{3}^{2}\right)^{1 / 2}, \\
& W=r\left(\beta^{2}-k^{2} n_{3}^{2}\right)^{1 / 2}, \\
& \Delta=\left(n_{2}^{2}-n_{3}^{2}\right) / 2 n_{2}^{2},
\end{aligned}
$$

where $n_{2}$ is the index of the fiber cladding, $n_{3}$ is the index of surrounding medium, $\beta$ is the propagation constant, $\lambda$ is the optical wavelength $(1550 \mathrm{~nm})$ and $k=2 \pi / \lambda$.

The power at the output ports is given by

$$
\begin{aligned}
& P_{3}=P_{0} \cos ^{2}(C L), \\
& P_{4}=P_{0} \sin ^{2}(C L),
\end{aligned}
$$

where $P_{0}$ is the input port power at port 1 and $L$ is the interaction length.

For a practical application, the device should be operated in the linear slope region of the sinusoidal response, in order to provide a linear relation between RI variations and the transmitted signal. For the experiments reported here, the coupler ratio is adjusted to obtain a zero normalized transmission, which corresponds to a 50:50 coupling ratio, when a drop of deionized water is deposited over the coupler waist $\left(\mathrm{n}_{\text {water }}=1.333\right)$. Additionally, we monitored the wavelength shift of the point with zero normalized transmission. Thus, the sensor response can also be codified in wavelength.
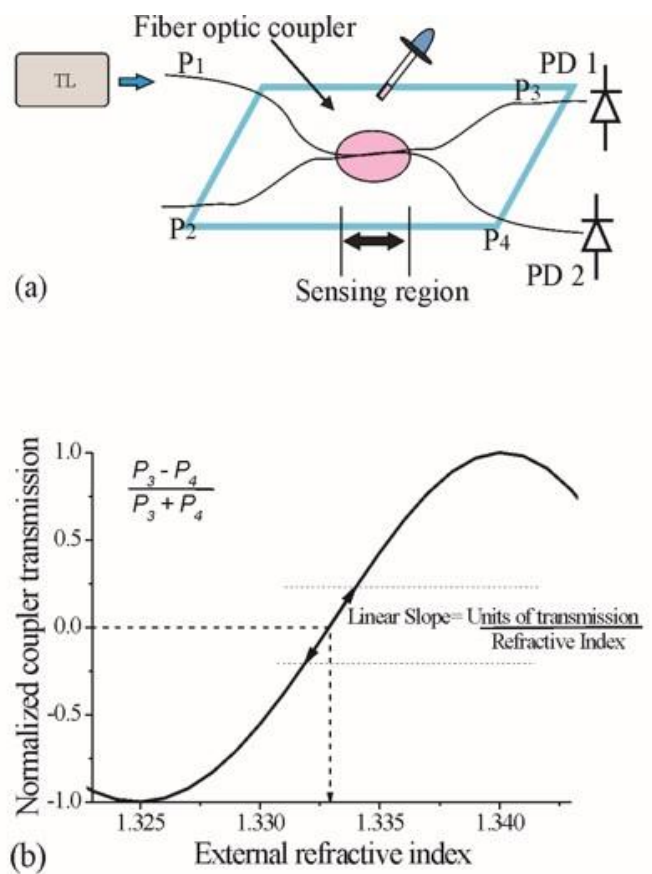

Fig. 2. (a) Schematic diagram of the experimental setup. (b) Numerical simulation of the normalized coupler transmission as a function of the external refractive index.

For the first set of experiments, the coupler is fabricated following the pulling process described in section II (see Fig. 1), with exponential profiles, but trying to obtain a device with the shortest waist length as possible. The pulling process is stopped at $17 \mathrm{~mm}$ of elongation, resulting in a coupler with 5 $\mu \mathrm{m}$ waist cross-section, and a waist length of about $1.5 \mathrm{~mm}$. The response of the sensor was investigated using two sets of calibrated sugar solutions. The first set had mass percent concentrations from 0 to $10 \%$, while the second set was prepared with concentrations from 0 to $1 \%$. The corresponding RI values were obtained from [44], [45], and all measurements were performed at room temperature. Fig. 3 shows the characterization of the sensor. Fig. 3(a) shows the spectral response for different sugar concentration solutions in the range from 0 to $10 \%$, corresponding to a RI in range from 1.333 to 1.349 , respectively. For this particular coupler, the zero normalized transmission for deionized water was obtained at the wavelength of $1542.9 \mathrm{~nm}$. Fig. 3(b) shows the relationship between normalized coupler transmission and 
sugar concentration at $1542.9 \mathrm{~nm}$. As it can be observed, the response shows a linear response for lower values of sugar concentration, but for higher values the sinusoidal expected response shows up, reaching its maximum value for a sugar concentration of $\sim 8 \%$. In the linear region, i. e., for sugar concentration in the interval $[0,8] \%$, the measured sensitivity is 0.10 units of normalized transmission per sugar concentration. The detection limit is determined by the noise signal level, which is originated by laser intensity fluctuations and noises associated from electronic circuits. In our experiments, the noise is reduced by averaging over a period of 1 minute at the oscilloscope, and the measured amplitude noise (AN) is 0.0016 units of normalized transmission. Therefore, the detection limit for this coupler is 0.016 weight percent of sugar solution. Taking into account the RI of a sugar solution, the RI detection limit is $2.4 \times 10^{-5}$ RIU.
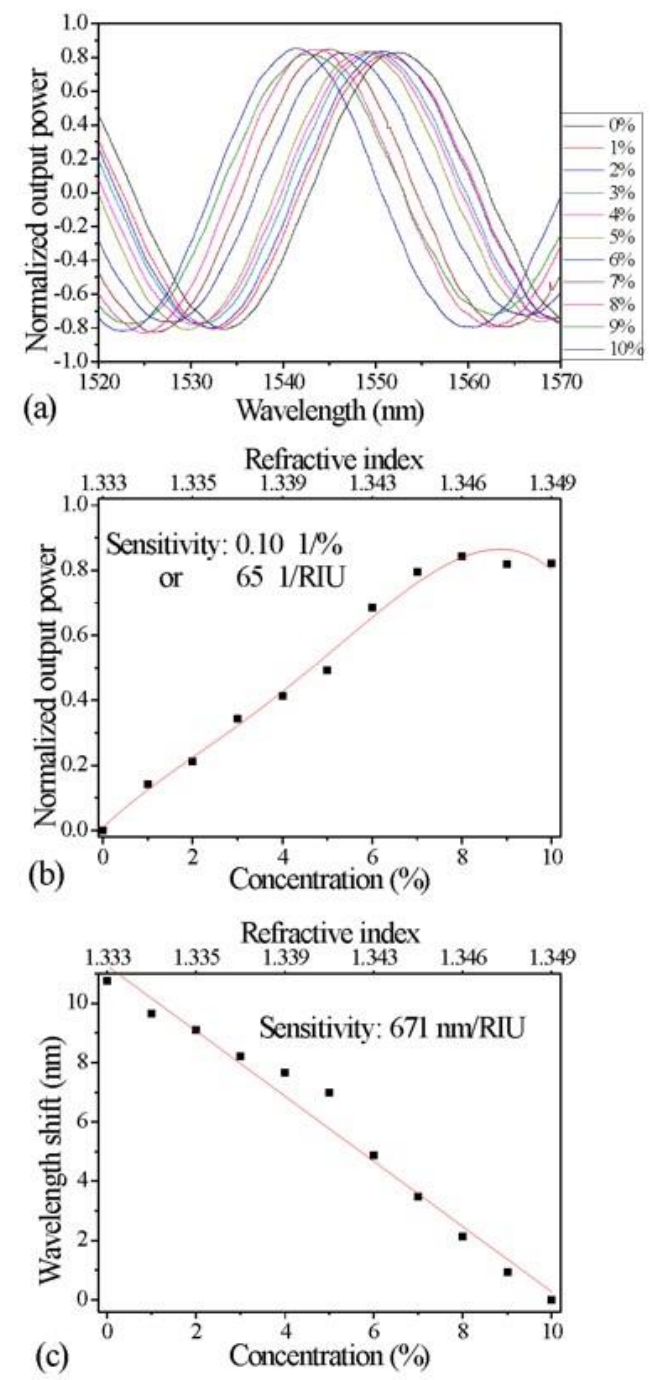

Fig. 3. Spectral and transmission responses of the overcoupled fiber coupler as a function of sugar concentration solutions in a range from 0 to $10 \%$. (a) Spectral response of the fused fiber coupler. (b) Normalized power transmission. (c) Shift in wavelength of zero normalized transmission.

The sensor can be wavelength-encoded by measuring the wavelength shift of the zero normalized transmission point as a function of sugar concentration, as shown in Fig. 3(c). It can be observed a linear response for the whole set of solutions, covering a relatively large range of RI, with a sensitivity of $671 \mathrm{~nm} / \mathrm{RIU}$. For estimation of wavelength resolution, we can assume that the amplitude noise is the actual limiting parameter, which gives a $\delta \lambda_{\min }=(\Delta \lambda / 2 \pi) \times \mathrm{AN}=9.7 \mathrm{pm}$, taking into account that the spectral response is sinusoidal and its period $\Delta \lambda$ is $38 \mathrm{~nm}$ for the present device. Thus, the estimated resolution is $1.4 \times 10^{-5} \mathrm{RIU}$. The free spectral range (FSR), which is the wavelength difference between two adjacent zero normalized transmission points, is measured as $16 \mathrm{~nm}$ from $\Delta \lambda$. To avoid ambiguity in experiments, the spectral range available for refractive index measurements is limited to be around $\Delta \lambda / 2 \mathrm{~nm}$ in wavelength shift.

With the objective of further improving in the coupler sensitivity, we fabricated a longer device with the same $5 \mu \mathrm{m}$ waist cross-section. The coupler was fabricated with an overall length of $19.5 \mathrm{~mm}$ and a waist length of $2.5 \mathrm{~mm}$. The sensitivity measurements were performed at the optical wavelength of $1557.7 \mathrm{~nm}$, which corresponds to the point of zero normalized transmission in deionized water. The spectral and transmission response for the sensor is depicted in the Fig. 4 for sugar concentration solutions in a range between 0 and 10\%. Fig. 4(a) shows the coupler spectral response for different sugar solutions, whereas the power transmission response is depicted in Fig. 4(b). At low sugar concentrations (between 0 and $1 \%$ ), the linear transmission slope is 0.442 units of normalized transmission per unit of sugar concentration (corresponding to 316 units of normalized transmission per RIU). Compared with the previous result, a significant improvement in sensitivity was obtained $(\times 4.6)$. The estimated detection limit is $3.6 \times 10^{-3}$ weight percent of sugar concentration, corresponding to a minimum detectable RI change of $5.06 \times 10^{-6}$ RIU. If we compare this result with recently reported papers [31], [37], this experimental result can be considered within the best results reported in the framework of fiber coupler based RI sensors whose operation relies on power transmission measurements. Fig. 4(c) shows the wavelength shift of the zero normalized transmission point as a function of sugar concentration. The experimental results show an average slope of $1029 \mathrm{~nm} / \mathrm{RIU}$ over the entire RI range from 1.333 to 1.349 . There is an improvement of $(\times 1.5)$ if we compare with the previous result. It should be pointed out that by increasing the sensitivity of this type of sensor, we will simultaneously reduce the FSR, as it happens in other interferometric-based sensors. 

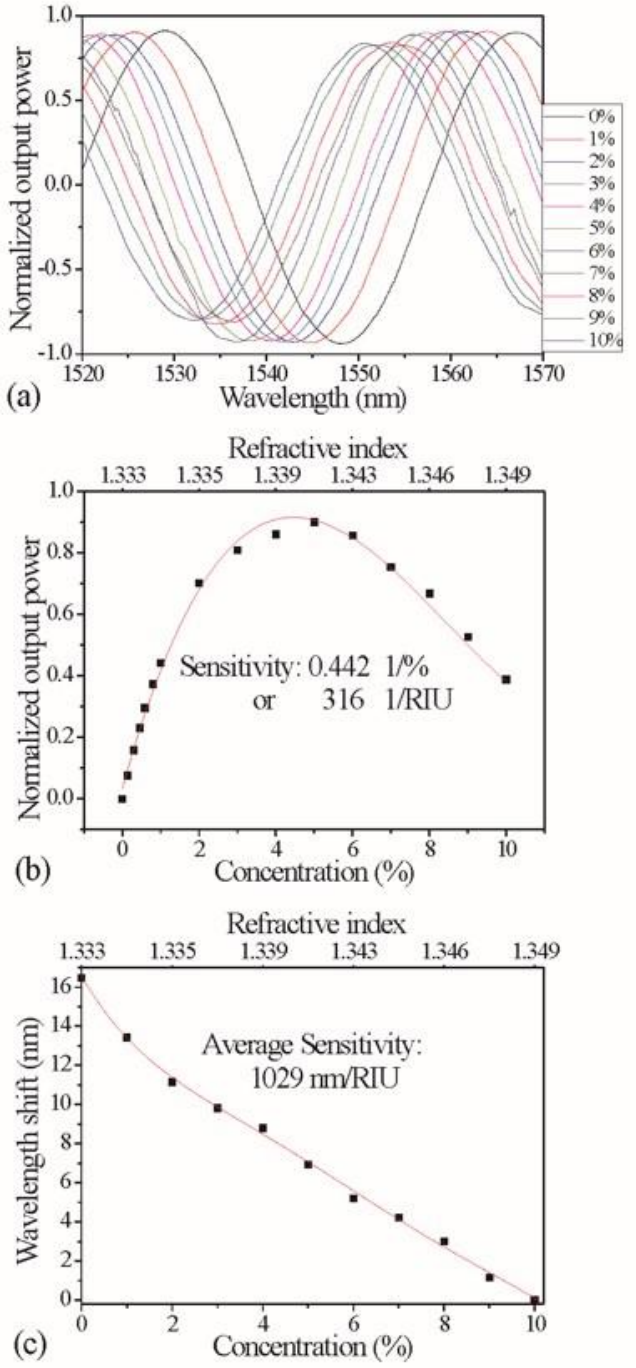

Fig. 4. Characterization of the $19.5 \mathrm{~mm}$ long fiber coupler as a function of sugar concentration: (a) Spectral response of the fused fiber coupler; (b) Normalized power transmission; (c) Wavelength shift of the zero normalized transmission point.

From the curve depicted in Fig. 4(c), it is clear that the device exhibits a higher sensitivity in the range from 0 to $1 \%$ of sugar concentration. Therefore, new measurements were performed within this range of sugar concentration, which correspond to RI variations ranging from 1.333 to 1.3344 . As it is shown in Fig. 5, experimental results demonstrate a linear response at low concentrations with a sensitivity of 2,171 $\mathrm{nm} / \mathrm{RIU}$, being the corresponding resolution in this range $4.4 \times 10^{-6}$ RIU.

Compared with other approaches in the literature, the sensitivity and resolution of the sensor, i. e., 2,171 nm/RIU and $\sim 5 \times 10^{-6}$ RIU, are comparable to that of fiber-optic surface plasmon sensors [46]-[49], side-polished fiber-optic sensors [50], and modal interferometers based on integrated waveguides [10]. Besides this, our proposed device maintains the advantages of compensation for power fluctuations, simple structure, compact size and easy fabrication. Additionally, the combination wavelength and power measurements enables the operation of the sensor in a large range of RI values, preserving a high resolution.

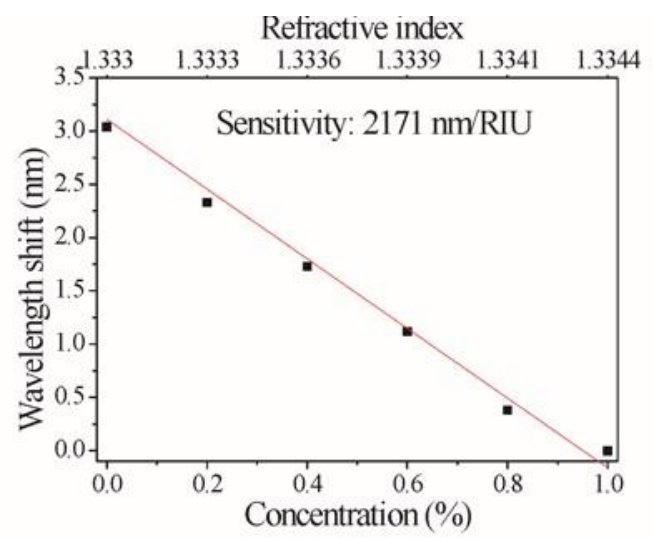

Fig. 5. Wavelength shift of zero normalized transmission point, for the 19.5$\mathrm{mm}$ long fiber coupler, as a function of sugar concentration between 0 to 1 $\%$.

In addition, our experimental results provide useful information for future optimization of fiber coupler based RI sensors. Our present results have not explored the differences between working with weakly coupled fibers, as the one depicted in the image of Fig. 1(c), and strongly fused fibers. Since the sensor response depends on the difference between the evanescent fields of the even and odd supermodes of the coupler, a fine control of the fused region will give an extra degree of freedom to improve de performance of the sensor.

\section{CONCLUSIONS}

In summary, we have investigated the optimization of fused biconical fiber couplers for the detection of small changes of RI of aqueous solutions. A significant improvement is obtained by operating the coupler in cycles well beyond their initial coupling cycles. The sensor can be operated either as amplitude-encoded or wavelength-encoded sensor, with sensitivities of 316 units of normalized transmission per RIU and $2,171 \mathrm{~nm} / \mathrm{RIU}$, and a detection limit as low as $5 \times 10^{-6}$ RIU, comparable to some of the best reported values for plasmon and integrated modal interferometers based sensors. However, our proposal exhibits some interesting advantages: the possibility of combining wavelength and power measurements in order to provide large RI range with high resolution, compensation for power fluctuations and easy fabrication. Further improvements could be achieved by optimizing the fused coupling region.

\section{ACKNOWLEDGMENT}

This investigation has been financially supported by CONACyT grant 222476. 


\section{REFERENCES}

[1] K. T. V Grattan, and T. Sun, "Fiber optic sensor technology: an overview," Sens. Actuator, A, vol. 82, no. 1-3, pp. 40-61, May. 2000.

[2] P. Lu, L. Men, K. Sooley, and Q. Chen, "Tapered fiber Mach-Zehnder interferometer for simultaneous measurement of refractive index and temperature," Appl. Phys. Lett, vol. 94, no. 13 pp. 131110-1-131110-3, Apr. 2009.

[3] K. T. Kim and K. H. Park, "Fiber-optic temperature sensor based on single mode fused fiber coupler," J. Opt. Soc. Korea, vol. 12, no. 3, pp. 152-156, Sep. 2008.

[4] Z. He, and K. Hotate, "Distributed fiber-optic stress-location measurement by arbitrary shaping of optical coherence function," $J$. Lightwave Technol, vol. 20, no. 9, pp. 1715-1723, Sep. 2002.

[5] K. Hotate, X. L. Song, and Z. He, "Stress-location measurement along an optical fiber by synthesis of triangle-shaped optical coherence function," IEEE Photonics Technol. Lett, vol. 13, no. 3, pp. 233-235, Mar. 2001.

[6] C. J. Lin, and F. G. Tseng, "A micro Fabry-Perot sensor for nano-lateral displacement sensing with enhanced sensitivity and pressure resistance," Sensor Actuator, A, vol. 113, no. 1, pp. 12-19, Jun. 2004.

[7] Y. Gong, T. Zhao, Y. Rao and Y. Wu, "All fiber curvature sensor based on multimode interference," IEEE Photonics Technol. Lett, vol. 23, no. 11, pp. 679-681, Jun. 2011

[8] S. Otsuki, K. Adachi, and T. Taguchi, "A novel fiber-optic gas-sensing configuration using extremely curved optical fibers and an attempt for optical humidity detection," Sens. Actuator, B. vol. 53, no. 1-2, pp. 9196, Nov. 1998.

[9] S. Zhang, W. Zhang, S. Gao P. Geng, and X. Xue, "Fiber-optic bending vector sensor based on Mach- Zehnder interferometer exploiting lateraloffset and up-taper," Opt. Lett, vol. 37, no. 21, pp. 4480-4482, Nov. 2012

[10] K. E. Zinoviev, A. B. González-Guerrero, C. Domínguez, and L. M. Lechuga, "Integrated bimodal waveguide interferometric biosensor for label-free analysis," J. Lightwave Technol, vol. 29, no. 13, pp. 19261930, Jul. 2011.

[11] T. Zhu, D. Wu, M. Liu, and D. W. Duan, "In-line fiber optic interferometric sensors in single-mode fibers," Sensors, vol. 12, no. 8, pp. 10430-10449, Aug. 2012.

[12] O. Esteban, M. Cruz-Navarrete, A. Gonzales-Cano, and E. Bernabeu, "Measurement of the degree of salinity of water with a fiber-optic sensor," Appl. Opt, vol. 38, no. 25, pp. 5267-5271, Sep. 1999.

[13] B. D. Gupta, H. Dojeda, and A. K. Tomar, "Fiber-optic evanescent field absorption sensor based on a U- shaped probe," Opt. Quantum Electron. vol. 28, no. 11, pp. 1629-1639, Nov. 1996.

[14] R. G. Lamont, D. C. Johnson, and K. O. Hill, "Power transfer in fused biconical-taper single-mode fiber couplers: dependence on external refractive index," Appl. Opt, vol. 24, no. 3, pp. 327-332, Feb. 1985.

[15] F. P. Payne, T. Finegan, M. S. Yataki, R. J. Mears, and C. D. Hussey, "Dependence of fused taper couplers on external refractive index," Electron. Lett, vol. 22, no. 22, pp. 1207-1209, Feb. 1986.

[16] K. Li, T. Zhang, G. Liu, N. Zhang, M. Zhang and L. Wei, "Ultrasensitive optical microfiber coupler based sensors operating near the turning point of effective group index difference," Appl. Phys. Lett, vol. 109, no. 10 pp. 101101-1-101101-4, Sep. 2016.

[17] L. Mao, S. Pu, D. Su, Z. Wang, X. Zeng, M. Lahoubi, "Magnetic field sensor based on cascade microfiber coupler with magnetic fluid," $J$. Appl. Phys, vol. 120, no. 9, pp. 093102-1-093102-6, Sep. 2016.

[18] J. Zhang, L. Shi, S. Zhu, X. Xu and X. Zhang, "Modeling of a singlenotch microfiber coupler for high-sensitivity and low detection-limit refractive index sensing," Sensors, vol. 16, no. 5, pp. 672, May. 2016.

[19] S. Wang, H. Yang, Y. Liao, X. Wang and J. Wang, "High-sensitivity salinity and temperature sensing in seawater based on a microfiber directional coupler," IEEE Photonics J, vol. 8, no. 4, Aug. 2016.

[20] S. C. Yan, Y. Chen, C. Li, F. Xu, and Y. Q. Lu, "Differential twin receveing fiber-optic magnetic field and electric current sensor utilizing a microfiber coupler," Opt. Express, vol. 23, no. 7, pp. 9407-9414, Apr. 2015

[21] Y. Chen, Y. Semenova, G. Farrell, F. Xu, and Y. Q. Lu, "A compact Sagnac loop based on a microfiber coupler for twist sensing," IEEE Photonics Technol. Lett, vol. 27, no. 24, pp. 2579-2582, Dec. 2015.

[22] L. Luo, S. Pu, J. Tang, X. Zeng and M. Lahoubi, "Highly sensitive magnetic field sensor based on microfiber coupler with magnetic fluid," Appl. Phys. Lett, vol. 106, no. 19 pp. 193507-1-193507-5, May. 2015.
[23] Y. Chen, S. C. Yan, X. Zheng, F. Xu and Y. Q. Lu, "A miniature reflective micro-force sensor based on a microfiber coupler," Opt. Express, vol. 22, no. 3, pp. 2443-2450, Jan. 2014.

[24] K. T. Kim, K. J. Cho, K. Im, S. J. Baik, C. H. Lee, and J. Lee, "High sensitivity refractive index sensor based on a wet-etched fused fiber coupler," IEEE Sens. J, vol. 11, no. 7, pp. 1568-1572, Jul. 2011.

[25] L. Bo, P. Wang, Y. Semenova, and G. Farrell, "High sensitivity fiber refractometer based on an optical microfiber coupler," IEEE Photonics Technol. Lett, vol. 25, no. 3, pp. 228-230, Feb. 2013.

[26] S. Pu, L. Luo, J. Tang, L. Mao, and X. Zeng, "Ultrasensitive refractiveindex sensor based on tapered fiber coupler with Sagnac loop," IEEE Photonics Technol. Lett, vol. 28, no. 10, pp. 1073-1076, May. 2016.

[27] C. R. Liao, D. N. Wang, X. He, and M. W. Yang, "Twisted optical microfibers for refractive index sensing," IEEE Photonics Technol. Lett, 23, no. 13, pp. 848-850, Jul. 2011

[28] P. Wang, M. Ding, G. Brambilla, Y. Semenova, Q. Wu and G. Farrell, "High temperature performance of an optical microfibre coupler and its potential use as a sensor," Electron. Lett, vol. 48, no. 5, pp. 283-284, 2012.

[29] L. Bo, C. C. O’Mahony, Y. Semenova, N. Gilmartin, P. Wang, and G. Farrell, "Microfiber coupler based label-free immunosensor," Opt. Express, vol. 22, no. 7, pp. 8150-8155, Apr. 2014.

[30] L. Bo, P. Wang, Y. Semenova, and G. Farrell, "Optical microfiber coupler based humidity sensor with a polyethylene oxide coating," Microwave Opt. Technol. Lett, vol 57, no. 2, pp. 457-459, Feb. 2015.

[31] H. Wei, Y. Zhu, and S. Krishnaswamy, "Optofluidic photonic crystal fiber coupler for measuring the refractive index of liquids," IEEE Photonics Technol. Lett, vol. 28, no. 1, pp.103-106, Jan. 2016.

[32] D. K. C. Wu, K. J. Lee, V. Pureur, and B. T. Kuhlmey, "Performance of refractive index sensors based on directional couplers in photonic crystal fibers," J. Lightwave Technol, vol. 31, no. 22, pp. 3500-3510, Nov. 2013.

[33] D. K. C. Wu, B. T. Kuhlmey, and B. J. Eggleton, "Ultrasensitive photonic crystal fiber refractive index sensor," Opt. Lett, vol. 34, no. 3, pp. 322-324, Feb. 2009.

[34] J. R. Guzman-Sepulveda, V. I. Ruiz-Perez, M. Torres-Cisneros, J. J. Sanchez-Mondragon, and D. A. May- Arrioja, "Fiber optic sensor for high-sensitivity salinity measurement," IEEE Photonics Technol. Lett, vol. 25, no. 23, pp. 2323-2326, Dic. 2013.

[35] K. J. Lee, X. Liu, N. Vuillemin, R. Lwin, S. G. Leon-Saval, A. Argyros, and B. T. Kuhlmey, "Refractive index sensor based on a polymer fiber directional coupler for low index sensing," Opt. Express, vol. 22, no. 14, pp. 17497-17507, Jul. 2014.

[36] M. V. Hernández-Arriaga, M. A. Bello-Jiménez, A. Rodríguez-Cobos, and M. V. Andrés, "Experimental investigation of fused biconical fiber couplers for measuring refractive index changes in aqueous solutions," IEEE Sens. J, vol. 16, no. 1, pp. 132-136, Jan. 2016.

[37] H. Tazawa, T. Kanie, and M. Katayama, "Fiber-optic coupler based refractive index sensor and its application to biosensing," Appl. Phys. Lett, vol. 91, no. 11, pp. 113901-1-113901-3, Sep. 2007.

[38] B. S. Kawasaki, K. O. Hill, and R. G. Lamont, "Biconical-taper singlemode fiber coupler," Opt. Lett, vol. 6, no. 7, pp. 327-328, Jul. 1981.

[39] T. A. Birks and Y. W. Li, "The shape of fiber tapers," J. Lightwave Technol, vol. 10, no. 4, pp. 432-438, Apr. 1992.

[40] F. P. Payne, C. D. Hussey and M. S. Yataki, "Polarisation analysis of strongly fused and weakly fused tapered couplers," Electron. Lett, vol. 21, no. 13, pp. 561-563, Jun. 1985.

[41] H. C. Chang, T. H. Lin and T. L. Wu, "Accurate coupling coefficients for fiber couplers with weakly fused cross sections," Appl. Opt, vol. 34, no. 27, pp. 6168-6171, Sep. 1995.

[42] R. Ismaeel, T. Lee, B. Oduro, Y. Jung, and G. Brambilla, "All-fiber fused directional coupler for highly efficient spatial mode conversion," Opt. Express, vol. 22, no. 10, pp. 11610-11619, May. 2014.

[43] A. Ankiewicz, A. W. Snyder, and X. H. Zheng, "Coupling between parallel optical fiber cores-Critical examination," J. Lightwave Technol, vol. LT-4, no. 9, pp. 1317-1323, Sep. 1986.

[44] W. M. B. M. Yunus and A. B. A. Rahman, "Refractive index of solutions at high concentrations," Appl. Opt, vol. 27, no. 16, pp. 33413343, Aug. 1988.

[45] L. Jiang, L. Zhao, S. Wang, J. Yang, and H. Xiao, "Femtosecond laser fabricated all-optical fiber sensors with ultrahigh refractive index sensitivity: modeling and experiment," Opt. Express, vol. 19, no. 18, pp. 17591-17598, Aug. 2011 
[46] R. Slavík, J. Homola, and J. Ctyroký, "Miniaturization of optic surface plasmon resonance sensor," Sens. Actuators, B, vol. 51, no. 1-3, pp. 311315, Aug. 1998.

[47] J. Homola, H. Vaisocherová, J. Dostálek, and M. Piliarik, "Multi-analyte surface plasmon resonance biosensing," Methods, vol. 37, no. 1, pp. 2636, Sep. 2005.

[48] M. Piliarik, M. Vala, I. Tichý, and J. Homola, "Compact and low cost biosensor based on novel approach to spectroscopy of surface plasmons," Biosens. Bioelectron, vol. 24, no. 12, pp. 3430-3435, Aug. 2009.

[49] H. S. Jang, K. N. Park, C. D. Kang, J. P. Kim, S. J. Sim, and K. S. Lee, "Optical fiber SPR biosensor with sandwich assay for the detection of prostate specific antigen," Opt. Commun, vol. 282, no. 14 pp. 28272830, Jul. 2009.

[50] H. Y. Lin, W. H. Tsai, Y. C. Tsao, and B. C. Sheu, "Side-polished multimode fiber biosensor based on surface plasmon resonance with halogen light," Appl. Opt, vol. 46, no. 5, pp. 800-806, Feb. 2007.

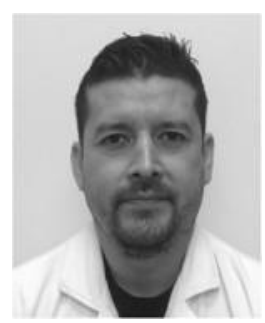

M. V. Hernández-Arriaga was born in Rioverde, México, in 1979. He received his M.Sc. degree in applied sciences from the Instituto de Investigación en Comunicación Óptica (IICO), at the Universidad Autónoma de San Luis Potosí (UASLP). He is currently pursuing the Ph.D. degree in applied sciences at the Instituto de Investigación en Comunicación Óptica (IICO). His research interest includes the development of optical fiber devices and fiber lasers.

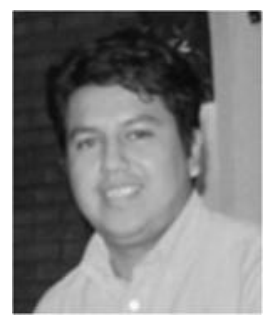

Miguel. Bello-Jiménez was born in Papantla, México, in 1981. He received his Ph.D. degree in optics from the National Institute for Astrophysics, Optics and Electronics (INAOE), Puebla, México, in 2010, and his electronics and communication engineer degree from the Universidad Veracruzana in 2003.

In 2010 he joined the Departamento de Física Aplicada y Electromagnetismo at the Universidad de Valencia (Spain) for two years, where he was engaged in the research on acoustooptic effects and actively mode-locked fiber lasers. Currently, he is Profesor-Investigador at the Instituto de Investigación en Comunicación Óptica (IICO) in the Universidad Autónoma de San Luis Potosí (UASLP). His current research interests include nonlinear effects, fiber optics, and fiber lasers.

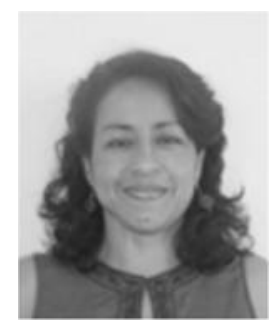

A. Rodríguez-Cobos was born in San Luis Potosí, México, in 1968. She received the B. S. in physics from the Universidad Autónoma de San Luis Potosí (UASLP), SLP, México, in 1993. The M.S. degree in Optics from the Centro de Investigación Científica de Estudios Superiores de Ensenada (CICESE), Ensenada, México, in 1995 and she received the $\mathrm{Ph}$. D degree in Optoelectronics from the Institut National Polytechnique de Grenoble, Grenoble, France, in 2001.

From 1995-1997, she was a Research Assistant with the CICESE Optics Laboratory. Since 2002 she has been a Professor-Researcher in the Instituto de Investigación en Comunicación Óptica, UASLP , México. Her research interest include the development of integrated optics devices, the applications of fiber optics devices and the technological application of photochromic polymer films. She is member of Mexican Society of Physics since 1995.

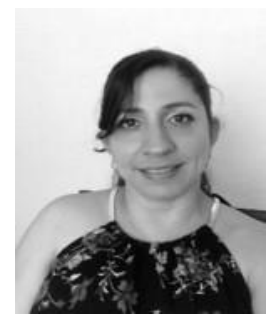

R. López-Estopier received the MS and $\mathrm{Ph} . \mathrm{D}$. degree in electronics from the National Institute for Astrophysics, Optics and Electronics (INAOE), Puebla, México, in 2010. In 2012, she joined the University of Valencia as a postdoctoral fellow researcher at the department of applied physics. In 2014 she joined the IICO-UASLP commissioned by CONACyT as a research fellow. Her research interests include semiconductors, silicon rich oxide, cathodoluminescence, photoluminescence, optoelectronic devices, and characterization of materials.

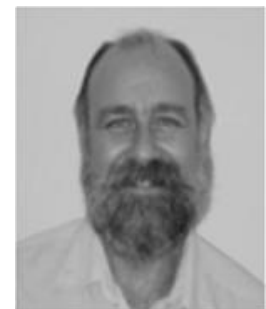

M. V. Andrés was born in Valencia (Spain) in 1957. He received the Licenciado en Física degree in 1979 and the Doctor en Física (Ph.D.) degree in 1985, both from the Universidad de Valencia (Spain). From 1983 he served successively as Assistant Professor and Lecturer in the Departamento de Física Aplicada of the Universidad de Valencia. At present, he is Professor of Applied Physics at the Departamento de Física Aplicada and member of the Instituto de Ciencia de los Materiales, both at the Universidad de Valencia.

Until 1984 he was engaged in research on microwave surface waveguides. From 1984 to 1987 he was visiting for several periods the Department of Physics, University of Surrey (U.K.), as a Research Fellow, working on fiber sensors. In 1987, he founded the Optical Fiber Laboratory group at the Universidad de Valencia. His current research interests include waveguide theory (photonic crystal fibers and inhomogeneous waveguides) and optical fiber devices and systems for microwave photonics, all-fiber lasers (active Qswitched and mode-locked fiber lasers) and sensor. 